\title{
Duración del tratamiento que se brinda en el Hospital Diurno a adultos con esquizofrenia y su relación con las habilidades psicosociales ${ }^{1}$
}

Institución: Hospital Nacional Psiquiátrico

Pbro. Manuel Antonio Chapuí y Torres

Programa CIEBE-CR

\section{COMO CITAR}

Román, V., Cantillo, Y., Salazar, F. (Abril, 2014) Duración del tratamiento que se brinda en el Hospital Diurno a adultos con esquizofrenia y su relación con las habilidades psicosociales. Rev. Actual de Costa Rica, 26, 1-9. Recuperado de: <http://www.revenf.ucr.ac.cr/esquizofrenia.pdf> ISSN 1409-4568

\section{RESUMEN}

El objetivo de este artículo es presentar los resultados de una investigación secundaria acerca del tiempo requerido para brindar tratamiento en un hospital diurno a personas adultas con esquizofrenia, con el fin de que desarrollen habilidades psicosociales. El Hospital Diurno del Hospital Nacional Psiquiátrico recibe actualmente personas tanto en fase aguda como crónica; las personas con una condición crónica cuentan con cinco o más años de estar participando de los programas que se ofrece en dicho centro hospitalario; a pesar de que algunas ya alcanzaron los objetivos propuestos en el momento de su ingreso, no han sido egresadas del centro, situación que limita de manera significativa la admisión de más personas que requieren de esta alternativa de atención especializada en salud mental y psiquiatría para el desarrollo de sus habilidades psicosociales. Ante este panorama, las investigadoras se formularon una pregunta en formato PICO (pacientes, intervención, comparación, resultados); posteriormente, se buscó información en distintas bases de datos sugeridas en el curso de Práctica Clínica Basada en la Evidencia, impartido por el Programa de Colaboración para la Investigación de Enfermería Basada en la Evidencia de Costa Rica (CIEBE-CR). Se detectó 37 documentos relacionados con el tema de los cuales sólo cinco cumplían criterios de calidad que establece el Critical Appraisal Skills Programme español (CASPe) para responder a la pregunta clínica. Las investigadoras concluyen que no existe suficiente evidencia para determinar cuál es el tiempo que requiere una persona con esquizofrenia en un hospital diurno para desarrollar sus habilidades psicosociales y ser egresado, hallazgo que motiva el desarrollo de investigación primaria que conduzca a la toma de decisiones respecto del tema.

Palabras clave: Esquizofrenia, habilidades-psicosociales, hospital-diurno.

\footnotetext{
${ }^{1}$ Fecha de recepción: 8 de agosto $2013 \quad$ Fecha de aceptación: 15 de noviembre 2013

${ }^{2}$ Enfermera en Salud Mental y Psiquiatría. Hospital Nacional Psiquiátrico. Correo electrónico: yani0818@gmail.com

${ }^{3}$ Enfermera en Salud Mental. Programa Rehabilitación Psicosocial, Hospital Diurno. Hospital Nacional Psiquiátrico. Correo electrónico: yorlecantillo@gmail.com

${ }^{4}$ Enfermera en Salud Mental. Subdirección en Educación. Hospital Nacional Psiquiátrico. Correo electrónico: floricelsm@gmail.com
} 


\title{
Duration of treatment provided in the Day Hospital for adults with schizophrenia and their relationship with psychosocial skills ${ }^{1}$
}

Institution: National Psychiatric Hospital

Pbro. Manuel Antonio Chapuí y Torres

CIEBE-CR Programme

\section{CITED}

\begin{abstract}
The aim of this paper is to present the results of secondary research about the time required to provide treatment in a day hospital for adults with schizophrenia, in order to develop psychosocial skills. The Day Hospital of the National Psychiatric Hospital currently receives people in both acute and chronic, and people with a chronic condition have five or more years of participating in the programs offered at that hospital; although some have already reached the goals set at the time of admission, have not been discharged of the hospital, a situation that significantly limits the admission of more people who need this care alternative specializing in mental health and psychiatry for the development of psychosocial skills. . Against this background, the researchers make a question in PICO format (patient, intervention, comparison, outcomes), Later, information was sought on various databases suggested in the course "Clinical Practice Evidence-Based" taught by the Program for Collaborative Research-Based Nursing Evidence of Costa Rica (CIEBE-CR). 37 documents related to the issue was detected of which just five met quality criteria. These were analyzed by applying the criteria set out in Critical Appraisal Skills Programme Spanish (CASPe) to answer the clinical question. The researchers conclude that there is insufficient evidence to determine the time it takes a person with schizophrenia in a day hospital to develop psychosocial skills and be discharged, so it is important to perform primary research, leading to the decisionmaking regarding theme.
\end{abstract}

Keywords: day-hospital, nursing, Schizophrenia, skills-psychosocial.

\footnotetext{
${ }^{1}$ Date of receipt: August 8, 2013

Date of acceptance: November 15, 2013

${ }^{2}$ Nurse in Mental Health and Psychiatric. National Psychiatric Hospital. E mail: yani0818@gmail.com

${ }^{3}$ Nurse in Mental Health and Psychiatric. Psychosocial Rehabilitation Program, Hospital Day. National Psychiatric Hospital. E mail: yorlecantillo@gmail.com

${ }^{4}$ Nurse in Mental Health and Psychiatric, Education Subdirectorate. National Psychiatric Hospital. E mail: floricelsm@gmail.com
} 


\section{INTRODUCCIÓN}

La salud mental se ha enfrentado a cambios de gran relevancia en la última década, debido a las características que presenta la población de acuerdo con el sistema de salud y el comportamiento epidemiológico, razón por la que la consulta de salud mental y la atención especializada en psiquiatría en Costa Rica no es ajena a tales transformaciones, por el contrario, la demanda de tal servicio se incrementa con el paso del tiempo. Esa nueva realidad implica el planteamiento de nuevas estrategias para la atención de las personas con un trastorno mental, así como de su familia y comunidad.

Los hospitales diurnos u hospitales de día representan una de esas estrategias de atención, debido a que proponen un proyecto terapéutico a las personas que llegan ahí cada día; por ende, se convierten en un espacio terapéutico, distinto del medio habitual o familiar, el cual-en ocasiones- genera cierta tensión, esfuerzo y participación más o menos activa para cumplir con el contrato terapéutico (Olivos, 1985).

Los centros de día, hospitales diurnos u hospitales de día, son establecimientos de servicios comunitarios formales de salud mental, esenciales para evitar las hospitalizaciones innecesarias. Estos servicios son un requisito previo imprescindible para dar el alta hospitalaria de los pacientes con trastornos mentales graves, para asegurar su rehabilitación y reintegración en la comunidad, prestar atención de seguimiento y apoyo a las familias y supervisar al personal sanitario de atención primaria (WHO, 2001 y 2003). Para la American Psychiatric Association (2002), estas alternativas, incluyendo la hospitalización parcial, "tienen la ventaja de evitar la disrupción de la vida del paciente, el tratamiento se realiza en un ambiente menos restrictivo y reduce el estigma asociado a la hospitalización psiquiátrica" (American Psychiatry Association, 2002, s.p.).

Los hospitales diurnos surgieron en 1932, según lo menciona Carballal (s.a.), como respuesta a las necesidades de atención. El primer hospital de día para la atención de usuarios con trastornos a nivel de psiquiatría surgió en Moscú y, de forma paralela a esta experiencia, aparecen las de Boyle en Chichester, Inglaterra, Woodall en Boston y Estados Unidos "siendo su implementación lenta hasta la década de 1960, la mayoría de los países industrializados reemplazan el hospital psiquiátrico por un nuevo modelo de atención en psiquiatría: la psiquiatría en la comunidad" (Olivos, 1985, p.7).

En Costa Rica, el Hospital Diurno del Hospital Nacional Psiquiátrico fue creado en 1969 y respondió a la iniciativa de un grupo de trabajadores de la salud que, partiendo de su experiencia profesional, ofrecieron asistencia psiquiátrica y psicológica múltiple y flexible a la población. Según Rodríguez (1991),

la ideología y recursos asistenciales del Hospital Diurno no apuntan tan solo a la simple supresión de los síntomas psicopatológicos, sino a configurar una dinámica de convivencia y relación terapéutica donde aquellas personas que se han visto avocadas a una situación de trastornos psíquicos estructuren o reestructuren su biografía, con una suficiente y saludable capacidad de autonomía y adaptación activa al entorno a través de un proceso terapéutico continuado y gradual que no suponga necesariamente una desvinculación de su medio social y familiar de pertenencia (p.29).

Actualmente, el Hospital Diurno atiende una población de 145 personas con trastorno mental, un $40 \%$ ha permanecido en el programa cinco años o más, a pesar de haber obtenido logros positivos reflejados en la Escala 


\section{Revista Electrónica Enfermería Actual en costa Rica}

de Evaluación para Personas con Trastorno Mental y de Conducta asociada a Enfermedad Mental y en los planes individuales de rehabilitación establecidos. Sin embargo, carecen de un apoyo real comunitario y externo al Hospital Nacional Psiquiátrico que le permitan reducir su permanencia o egreso del programa.

Lo anterior lleva a las investigadoras a generar una pregunta clínica de primera línea en formato PICO referente al tema que expone este artículo, cuya respuesta pueda conducir a elaborar estrategias de atención y toma de decisiones basadas en la evidencia científica.

El objetivo fue conocer cuánto tiempo requiere una persona con esquizofrenia ingresada en un hospital diurno para desarrollar las habilidades psicosociales necesarias para desenvolverse de forma autónoma e independiente en su comunidad.

\section{MATERIALES Y MÉTODOS}

Para efectuar la presente investigación, se empleó la metodología recomendada para llevar a cabo la práctica de Enfermería Basada en la Evidencia (EBE). Se considera la EBE como "un enfoque de toma de decisiones en el cual los clínicos usan la mejor evidencia disponible, en consulta con los pacientes, para decidir cuál es la opción que es más aceptable con lo mejor que tenga el paciente" (Urra et al 2010, p.2). Esta metodología establece cinco pasos:

1. Formulación de una pregunta de primera línea en el formato PICO.

2. Búsqueda de información científica disponible.

3. Análisis crítico de los datos.

4. Implementación de los resultados encontrados en el quehacer diario del profesional.

5. Evaluación de la implementación.

Con base en la necesidad sentida por las profesionales de enfermería y siguiendo el primer paso, se generó una pregunta en formato PICO de tipo intervención, la cual se desglosa de la siguiente manera:

- Tipo de paciente o la patología de la que surge la pregunta $(\mathrm{P})=$ personas con esquizofrenia.

- La intervención que queremos analizar (I) = tratamiento que se brinda en hospitales diurnos durante cinco años.

- La comparación con otra intervención (si procede) $(C)=$ tratamiento que se brinda en hospitales diurnos a corto plazo (2 años).

- Los resultados clínicos esperados $(\mathrm{O})=$ aumento en el desarrollo de habilidades psicosociales.

La pregunta formulada fue: En personas con esquizofrenia, ¿el tratamiento que se brinda en hospitales diurnos durante cinco años comparado con el tratamiento a corto plazo (2 años) aumenta el desarrollo de las habilidades psicosociales?

Seguidamente, se efectuó una revisión de artículos científicos en PubMed, LILACS, Medline Plus, Google Académico, Scielo y EBSCO Host, con el fin de recuperar la mayor cantidad de información. Se emplearon los siguientes descriptores: Schizophrenia AND day hospital, schizophrenia AND day hospital AND psycochosocial 


\section{Revista Electrónica Enfermería Actual en costa Rica}

skills, schizophrenia AND psycochosocial skills, benefits of attending AND day hospital, day hospital AND treatment, schizophrenia AND day center, benefits AND day hospital, day hospital treatment AND schizophrenia, center day OR day hospital.

En la búsqueda se recuperó 37 documentos de los cuales 31 fueron excluidos, debido a factores metodológicos; es decir, por lo que no alcanzaron los criteros de calidad para el análisis crítico de la información; por lo tanto, solo cinco documentos sirvieron para ser analizados.

La siguiente etapa consistió en el análisis crítico de la calidad de la información de los cinco documentos seleccionados a los que se aplicó la plantilla Critical Appraisal Skills Programme Spanish (CASPe), herramienta usada para el análisis crítico de la literatura científica (http://redcaspe.org.). Por último, con la mejor información recuperada y seleccionada se procedió a responder la pregunta PICO planteada.

En la cuarta etapa, que se refiere a la implementación de la evidencia, las investigadoras decidieron presentar los resultados a las autoridades tanto médicas como de enfermería del nosocomio, para determinar diferentes acciones en conjunto. Posteriormente se retomará la etapa de evaluación.

\section{Consideraciones éticas}

Dentro de las consideraciones éticas, para cada material que se utilizó en el desarrollo de la presente investigación, se respetaron los derechos de autor, incorporando a lo largo del texto las citas bibliográficas correspondientes. En cuanto al tratamiento de los documentos recolectados (datos), su manejo concuerda con la metodología de Práctica de Enfermería Basada en la Evidencia.

\section{RESULTADOS}

Los cinco estudios incluidos correspondían a información relacionada con el tratamiento y los beneficios que reciben las personas con esquizofrenia en los hospitales de día u hospitales diurnos, información de la que se desprendió la siguiente evidencia:

1. Efficacy of psychosocial rehabilitation program: The RFS experience (Rupasri, C., Ramaprasad, D., S. Kalynasundaram y Rao, S. 2011). Estudió la eficacia del programa de rehabilitación de la Sociedad Richmond Fellowship (RFS) "a mitad de camino casa", en Estados Unidos de América: concluyeron que las personas con discapacidades psiquiátricas necesitan apoyo para su recuperación y se pueden beneficiar de programas de rehabilitación. Cuando las familias tienen dificultades para practicar estas estrategias, los programas residenciales pueden ayudarles a conseguir que sus seres queridos regresen a su hogar, además de reducir la carga del cuidador.

2. Application in Mexico of Psychosocial Rehabilitation with Schizophrenia Patients (Valencia et al, 2010). Demostró que los programas de rehabilitación psicosocial, dirigidos a pacientes con esquizofrenia y desarrollados en Estados Unidos e Inglaterra, también son eficaces en países en desarrollo y con una considerable diferencia cultural, como lo es la Ciudad de México. Solo se hicieron pequeñas modificaciones para que los tratamientos fueran mejor aceptados por la cultura mexicana. 
3. A Meta-Analysis of Controlled Research on Social Skills Training for Schizophrenia (Kurtz, M., Mueser, K., 2008). La investigación pretendía construir un metaanálisis (a), incluyendo solo los ECA con muestras en las que la mayoría de los clientes tuvieran diagnósticos de esquizofrenia o trastorno esquizoafectivo; investigaban el impacto de las variables de entrenamiento en habilidades sociales, activos frente al tratamiento por uso habitual de las habilidades sociales, condiciones de control, características de los participantes en los resultados observados, y la categorización y evaluación de resultados. Concluye en que la razón principal de los programas de entrenamiento de habilidades sociales en la esquizofrenia mejoran el funcionamiento psicosocial, apoyan la mejora funcional en el ajuste social y la vida independiente, además de los síntomas negativos, que están fuertemente asociadas al deterioro de la función psicosocial, lo cual muestra una fuerte evidencia para la generalización de intervenciones en el entorno de la formación a los ámbitos más complejos de funcionamiento diario. También ayudan a los clientes a alcanzar sus metas personales a través de la práctica de comportamientos eficaces, cuyo fin es reforzar la autoeficacia y la voluntad de utilizar las habilidades existentes. El análisis reveló efectos significativos en otros síntomas psiquiátricos y las recaídas fueron efectos más distales. No obstante, no está claro si la respuesta más pobre en los síntomas negativos de los clientes más antiguos refleja una característica de la muestra o el grado en que los programas de entrenamiento de habilidades sociales no abordan las necesidades de los clientes más antiguos.

4. Centro de día para enfermedades mentales (Catty et al, 2008). El estudio determinó que los centros de día para las personas con enfermedades mentales graves y sus cuidadores, -cuando los recursos son limitadospueden ser la única manera de asegurar la equidad en la prestación de los servicios de salud.

5. Day hospital outpatient care for people with schizophrenia (Review) (Shek, E., Shansis, F., Marshall, Crowthers, R. y Tyrer, P., 2009). Destaca que las personas con esquizofrenia, trastornos similares a la esquizofrenia y los trastornos bipolares pueden evitar el ingreso en la atención hospitalaria, cuando participan en los hospitales de día. De igual forma, muestran la necesidad de mejorar la calidad de los resultados y realizar nuevos estudios en relación con este tema.

\section{DISCUSIÓN}

En el Hospital Diurno del Hospital Nacional Psiquiátrico se ha evidenciado, a través de los programas de intervención y seguimiento multidisciplinario, que las personas con trastorno mental que asisten al menos una vez por semana tienen una reducción de los predictores clínicos de recaídas, por lo que logran un eficiente manejo de los predictores psicosociales.

Asimismo, se ha observado que "el número de hospitalizaciones son reducidos, y cuando se dan son en unidades agudas, y de pocos días, lo cual también ha sido confirmado en países como Inglaterra, Unión Soviética y Norteamérica" (Naranjo, 2001, p.6).

Sin embargo, en los estudios revisados se demuestra la efectividad y beneficios de los hospitales diurnos, en relación con la accesibilidad y asequibilidad, son menos costosos. En lo referente a la aceptabilidad, el estigma y la consecuente discriminación son muy reducidas en comparación con los servicios especializados y en los hospitales psiquiátricos en particular reducen la probabilidad de que se produzcan violaciones de los derechos 
humanos, el aislamiento es menor que cuando reciben atención hospitalaria lejos de sus hogares y la atención integral asegura mejores resultados en cuanto al tratamiento.

Por otra parte, una de las debilidades que podría tener el Hospital Diurno es su ubicación dentro del mismo Hospital Psiquiátrico, según Naranjo (2001) "es altamente contraproducente instalar hospitales diurnos en hospitales psiquiátricos, ya que de esa manera son absorbidos por la dinámica de ellos, brindando pocas posibilidades de rehabilitación y de no estigmatización" (p.16).

En Costa Rica se ha observado que el perfil de funcionamiento de las personas con enfermedad mental que están demandando la atención en el Hospital Diurno es mayor en comparación con años anteriores, situación que ha convertido el programa en uno de preparación, acompañamiento y respiro familiar que favorece el reforzamiento y recuperación de habilidades que se orientan a la reincorporación laboral, social y académica. Por lo anterior es que los profesionales de salud han retomado esta experiencia y empezaron a reformular plazos para el logro de objetivos de acuerdo con los planes individuales establecidos, además de considerar que los plazos prolongados de atención obedecen a personas con limitaciones, en quienes los síntomas negativos y el deterioro son más evidentes, sin mencionar que carecen de una red de apoyo comunitaria.

Ninguna de las investigaciones analizadas se refiere al tiempo que la persona con esquizofrenia debe permanecer en los programas de los hospitales diurnos; sin embargo, en Chile, el Ministerio de Salud establece que los (as) usuarios (as) con enfermedad mental asistirán al hospital diurno por un periodo que va desde semanas hasta cuatro meses, considerando la realidad local y las necesidades del usuario (Ministerio de Salud, Chile, 2001). En la mayoría de estudios se enfatiza los beneficios en relación con la adquisición de habilidades psicosociales.

\section{CONCLUSIÓN}

No se encontraron estudios en personas adultas con esquizofrenia que demuestren el aumento en el desarrollo de habilidades psicosociales a corto plazo, en comparación con personas con este mismo trastorno en un lapso de cinco años; por consiguiente, es necesario desarrollar estudios que consideren los factores que influyen en la permanencia de la persona con trastorno mental en el hospital diurno, aún después de haber logrado los objetivos establecidos en su tratamiento.

\section{REFERENCIAS BIBLIOGRÁFICAS}

American Psychiatry Association. (2002). Clinical Resources; Schizofenia: Ch. III: Treatment principles and alternatives, G Treatment Settings; 1.c. Recuperado de www.psyc.org.

Carballal, C. (s.a.). Proyecto: El Papel de Enfermería en un hospital de Día de Salud Mental. Recuperado de http://www.psiquiatriasur.cl/portal/uploads/5_conociendo_el_hospital_de_dia_en_psiquiatria.doc

CASPe, Fichas de lectura crítica. Recuperado de http://redcaspe.org. 
Catty J.S., Bunstead Z., Burns T. y Comas A. Centros de día para enfermedades mentales graves (Revisión Cochrane traducida). En La Biblioteca Cochrane Plus, 2008 Número 4. Oxford: Update Software Ltd. Disponible en http://www.update-software.com.

Kurtz, M. y Mueser, K. (2008). A Meta-Analysis of Controlled Research on Social Skills Training for Schizophrenia. Journal of Consulting and Clinical Psychology, Vol 76 (3), Jun, 491-504.

Ministerio de Salud (2011). Orientaciones técnicas para el funcionamiento de los hospitales diurnos. Chile.

Naranjo, C. (2001). Hospital de Día en Psiquiatría. Evolución de la Experiencia Mundial y Estado de la Situación en Chile. Chile: Universidad de Santiago.

Olivos, P. (1985). Historia de los Hospitales Diurnos. Chile: Ministerio de Salud.

Rodríguez, J. (1991). Reseña Histórica del Hospital Nacional Psiquiátrico. Recuperado de hpcs.bvs.sa.cr/textos/doc42.pdfi

Rupasri, C., Ramaprasad, D., Kalynasundaram S. y Suryanarayana, R. (2011). Efficacy of psychosocial rehabilitation program: The RFS experience. Indian Journal of Psychiatry, 53(1)

Shek, E., Shansis, F., Marshall, Crowthers, R. y Tyrer, P. (2009). Day hospital outpatient care for people with schizophrenia. Cochrane Database of Systematic Reviews 2009, Issue 4. Art. No.: CD003240. DOI:

10.1002/14651858.CD003240.pub2.

Urra, E., Retamal, C., Tapia, C. y Rodríguez, M. (2010). Enfermería basada en la evidencia: qué es, sus características y dilemas. Investigación y Educación en Enfermería.

Valencia, M., Rascon, M.L., Juarez, F., Escamilla, R., Saracco, R. y Liberman, R.P. (2010). Application in Mexico of Psychosocial Rehabilitation with Schizophrenia Patients. Psychiatry 73(3) Fall 2010.

World Health Organization (2001). Mental health: new understanding, new hope. Ginebra, Organización Mundial de la Salud.

World Health Organization (2003). Organization of services for mental health. Ginebra, Organización Mundial de la Salud, 2003 (paquete de orientación sobre políticas y servicios de salud mental. 